\title{
Kinetic-energy systems, density scaling, and homogeneity relations in density-functional theory
}

\author{
Garnet Kin-Lic Chan and Nicholas C. Handy \\ Department of Chemistry, University of Cambridge, Lensfield Road, Cambridge CB2 1EW, United Kingdom
}

(Received 29 April 1998)

\begin{abstract}
We examine the behavior of the Kohn-Sham kinetic energy $T_{s}[\rho]$ and the interacting kinetic energy $T[\rho]$ under homogeneous density scaling, $\rho(\mathbf{r}) \rightarrow \zeta \rho(\mathbf{r})$. Using convexity arguments, we derive simple inequalities and scaling constraints for the kinetic energy. We also demonstrate that a recently derived homogeneity relation for the kinetic energy [S. B. Liu and R. G. Parr, Chem. Phys. Lett. 278, 341 (1997)] does not hold in real systems, due to nonsmoothness of the kinetic-energy functional. We carry out a numerical study of the density scaling of $T_{s}[\rho]$ using ab initio densities, and find it exhibits an effective homogeneity close to $5 / 3$. We also explore alternative reference systems for the kinetic energy which have fewer particles than the true $\mathrm{N}$-particle interacting system. However, we conclude that the Kohn-Sham reference system is the only viable choice for accurate calculation, as it contains the necessary physics. [S1050-2947(99)05903-X]

PACS number(s): 31.15.Ew
\end{abstract}

\section{INTRODUCTION}

Density-functional theory yields observables, such as the energy, as functionals of the density $\rho(\mathbf{r})[1]$. In recent years the Kohn-Sham version of density-functional theory [2], which offers a set of exact, self-consistent field equations, has become the principal computational method of molecular and condensed-matter physics. The main problem is that the form of the energy functional $E[\rho]$ is unknown.

In the development of new density functionals, there has been much interest in the behavior of energy components, when $\rho(\mathbf{r})$ undergoes some form of scaling. Most workers have focused on coordinate scaling [3], where $\rho(\mathbf{r})$ $\rightarrow \zeta^{3} \rho(\zeta \mathbf{r})$, i.e., electronic coordinate scaling: this effectively explores the effect of changing the external potential associated with $\rho(\mathbf{r})$, but not the particle number. One application of this formalism is in the construction of functionals, since constraining functionals to satisfy derived coordinate scaling requirements extends the effective range of validity of the data set used to parametrize the functionals [4]. A second application is to derive virial relations [3], where if a functional (such as the exchange energy $E_{x}[\rho]$ ), has a single polynomial dependence on $\zeta$, one can write down exact relations like $E_{x}[\rho]=-\int d \mathbf{r} \rho(\mathbf{r}) \mathbf{r} \cdot \nabla v_{x}(\mathbf{r})$, where $v_{x}(\mathbf{r})$ is the functional derivative of $E_{x}[\rho]$.

Here, we investigate a different type of scaling, homogeneous density scaling (from now on, simply "density scaling', ), where $\rho(\mathbf{r}) \rightarrow \zeta \rho(\mathbf{r})$. Through density scaling, we are changing both the particle number and the external potential associated with $\rho(\mathbf{r})$. We believe that density scaling may offer an approach to the derivation of exact conditions on functionals. Also, if functionals exhibit simple behavior with density scaling, we can expect to derive relations analogous to the virial relations of coordinate scaling theory. The latter aspect has previously been investigated in the recent work of Parr and Liu [5], and Liu and Parr [6], who proposed simple "homogeneity relations" for separate components of the energy.

In this study of density scaling, we focus on the kinetic energy (the interacting kinetic energy $T[\rho]$, as well as the Kohn-Sham kinetic energy $\left.T_{s}[\rho]\right)$. This is for two related reasons. First, we do not consider the problem of representing the kinetic energy in density-functional theory, a solved problem. This is because, although the Kohn-Sham kinetic energy functional $T_{s}[\rho]$ [2] gives a good representation of the kinetic energy, it is not a simple density functional, as it depends on an auxiliary set of orbitals $\phi_{i}(\mathbf{r})$. We wonder, as have many others [7-9], if it is not possible to find some simpler representation of the kinetic energy, which is a sufficiently accurate approximation in certain density regimes, to $T_{s}[\rho]$ or $T[\rho]$. Accordingly, knowledge of the density scaling behavior of the kinetic energy will aid in the construction of suitable alternative kinetic-energy functionals.

Second, from a different point of view, because the explicit form of the Kohn-Sham kinetic energy $T_{s}[\rho]$ is known, and can be calculated from any density using recently developed procedures [10], we can study the density scaling of $T_{s}[\rho]$ numerically. This information is interesting in its own right, and also, since $T_{s}[\rho]$ is a tight lower bound to $T[\rho]$, studying the scaling of $T_{s}[\rho]$ yields much information on the scaling of $T[\rho]$.

Our study proceeds as follows. Density scaling does not preserve normalization, but instead simultaneously scales the particle number. This requires us to consider kinetic energies and other quantities at noninteger particle numbers, for which the density matrix, rather than the wave function, becomes the object of interest in quantum mechanics. In Sec. II, we give the density-matrix definitions of $T[\rho], T_{s}[\rho]$, and other relevant density-functional quantities, for general particle numbers. In addition, we define the auxiliary quantity $T_{\zeta}[\rho]=T_{s}[\zeta \rho] / \zeta$, the $\zeta$-kinetic energy, which relates the density scaling of $T_{s}[\rho]$ to the particle number $(N)$ scaling of a generalized Kohn-Sham reference system. For $\zeta$ such that $N / \zeta$ is an integer, $T_{\zeta}[\rho]$ is a kinetic-energy functional that depends on $N / \zeta$ auxiliary orbitals, and is thus a possible candidate for a simpler kinetic-energy functional. For example, when $\zeta=N, T_{\zeta}[\rho]$ is the von Weizsäcker kinetic energy.

Next, in Sec. III, we examine density scaling of $T_{s}[\rho]$ and $T[\rho]$ from a formal point of view, using $T_{\zeta}[\rho]$ as an intermediary. The concepts of convexity and homogeneity are 
introduced. Using convexity, we present simple inequalities, such as

$$
\begin{gathered}
T_{s}[\zeta \rho] \geqslant \zeta T_{s}[\rho] \quad(\zeta>1), \\
T_{\zeta}[\rho] \leqslant T_{\zeta^{\prime}}[\rho] \quad\left(\zeta>\zeta^{\prime} \mid \zeta, \zeta^{\prime} \geqslant 1\right),
\end{gathered}
$$

which seem obvious, though we have not encountered them in the literature. We also highlight an important scaling constraint due to Lieb and Thirring [11]. Using these scaling conditions on $T_{s}[\rho]$ and $T[\rho]$, we demonstrate that the kinetic-energy homogeneity relation of Liu and Parr [6] cannot hold, and that simple homogeneity is not precise enough a concept to describe nonsmooth functionals. We introduce the concept of an effective homogeneity, for nonsmooth functionals.

Then, in Sec. IV, we study the density scaling of $T_{s}[\zeta \rho]$ with $\zeta$, numerically. We first compute the $\zeta$-kinetic energy $T_{\zeta}[\rho]$, from the $a b$ initio densities of the closed-shell spherical atoms $\mathrm{Be}, \mathrm{Mg}, \mathrm{Ne}$, and $\mathrm{Ar}$, and then calculate the corresponding $T_{s}[\zeta \rho]$ through a scaling relation. We briefly present our computational scheme, based on the inversion of the generalized Kohn-Sham orbital equations associated with $T_{\zeta}[\rho]$, through the method of Zhao-Morrison-Parr [12], though we relegate the full derivation of the orbital equations and Zhao-Morrison-Parr method to Appendix B. Our calculations verify the scaling conditions derived in Sec. III, and we further demonstrate that the Kohn-Sham kinetic energy $T_{s}[\rho]$ displays an effective homogeneity close to $5 / 3$. Also, we demonstrate, through $T_{\zeta}[\rho]$, that we cannot easily compute the kinetic energy unless we have the same number of reference orbitals as we have particles in the system.

Finally in Sec. V, we summarize our findings, and indicate some future directions.

\section{DEFINITIONS}

We first consider the definitions of the relevant quantities in this study, and how they encompass the operation of density scaling. Note that we assume all densities concerned are $N$ - and $v$-representable.

Following the work of Levy [13] and Lieb [14], we usually define energy density functionals through constrained minimizations. For example, we have, for the total energy $E[\rho]$ and Kohn-Sham kinetic energy $T_{s}[\rho]$ of an integer number of particles,

$$
\begin{gathered}
E[\rho]=\min _{\Psi \rightarrow \rho}\langle\Psi|\hat{T}+\hat{V}| \Psi\rangle+\int \rho(\mathbf{r}) v(\mathbf{r}) d \mathbf{r}, \\
T_{s}[\rho]=\min _{\Psi \rightarrow \rho}\langle\Psi|\hat{T}| \Psi\rangle,
\end{gathered}
$$

where $\hat{T}$ and $\hat{V}$ are the kinetic and potential operators, respectively, and $v(\mathbf{r})$ is the external potential. The interacting kinetic energy $T[\rho]$, is defined as $\left\langle\Psi_{m}|\hat{T}| \Psi_{m}\right\rangle$, where $\Psi_{m}$ minimizes Eq. (3). Note, that since the minimizing $\Psi$ in Eq. (4) is a Slater determinant of $N$ orbitals $\phi_{i}(\mathbf{r})$, we can write $T_{s}[\rho]$ in the familiar form, $T_{s}[\rho]=\min _{\Sigma_{i}^{N} \phi_{i}^{2} \rightarrow \rho} \Sigma_{i}^{N}$ $-\frac{1}{2}\left\langle\phi_{i}\left|\nabla_{i}^{2}\right| \phi_{i}\right\rangle$.
However, under the operation of density scaling, $\rho(\mathbf{r})$ $\rightarrow \zeta \rho(\mathbf{r})$, the number of particles changes from $N$ to $\zeta N$, where $N=\int \rho(\mathbf{r}) d \mathbf{r}$. Thus, as $\zeta$ is varied, we pass through densities with noninteger particle numbers, which the definitions (3) and (4) do not handle. How do we interpret this? No real isolated system can possess a noninteger particle number, and thus there is no unique interpretation. However, subsystems of larger systems may well have an average noninteger particle number [15], and such subsystems are described using density matrices, rather than wave functions.

Thus the natural context in which to treat noninteger particle numbers, in density-functional theory, is simply to extend the constrained search over the density-matrix operator $\hat{\Gamma}[16] . \hat{\Gamma}$ has the spectral representation

$$
\hat{\Gamma}=\sum_{M i} f_{M i}\left|\Psi_{M i}\right\rangle\left\langle\Psi_{M i}\right|,
$$

where $\Psi_{M i}$ is the $i$ th $M$-particle eigenfunction of a Hamiltonian, and $f_{M i}$ are occupations numbers which satisfy the conditions, $0 \leqslant f_{M i} \leqslant 1$, and $\Sigma_{M i} f_{M i}=1$. In terms of the density matrix, the expectation value of an operator $\hat{\Omega}$ is yielded by the linear operation $\operatorname{Tr}[\hat{\Gamma} \hat{\Omega}]$. Note that $\hat{\Gamma}$ may yield an average noninteger particle number.

Then, replacing the wave function by the density-matrix operator, the extended definitions for the energy functionals corresponding to Eqs. (3) and (4), which are valid for all particle numbers, become

$$
\begin{aligned}
& E[\rho]=\min _{\hat{\Gamma} \rightarrow \rho(\mathbf{r})} \operatorname{Tr}[\hat{\Gamma}(\hat{T}+\hat{V})]+\int \rho(\mathbf{r}) v(\mathbf{r}) d \mathbf{r} \\
& T_{s}[\rho]=\underset{\hat{\Gamma} \rightarrow \rho(\mathbf{r})}{\min } \operatorname{Tr}[\hat{\Gamma} \hat{T}],
\end{aligned}
$$

where $T[\rho]$ is given by $\operatorname{Tr}\left[\hat{\Gamma}_{m} \hat{T}\right]$, and $\hat{\Gamma}_{m}$ minimizes Eq. (6). At the integer densities used in this work, we shall assume that the density matrix (6), (7) and wave function definitions (3), (4) are identical. Similar generalizations of $E[\rho]$ in terms of $\hat{\Gamma}$ have been used previously by Mermin [17] and Perdew et al. [15].

Before proceeding, several points must be noted. First, although scaling $\rho(\mathbf{r}) \rightarrow \zeta \rho(\mathbf{r})$ is a linear procedure, the map of $\Gamma_{m} \rightarrow \rho(\mathbf{r})$ is highly nonlinear, and moreover, has a nontrivial dependence on $N[15,14]$. Thus, a priori, we do not expect the general energy functionals (6), (7) to have a simple behavior under density scaling. Second, we mention again that under density scaling the ground-state external potential associated with $\rho(\mathbf{r})$ (through the Hohenberg-Kohn theorem) changes in a nontrivial fashion. However, from Eqs. (6) and (7), we see that the external potential does not enter into the definitions of the kinetic energy, and thus will not concern us in this work.

In the case of the Kohn-Sham kinetic energy $T_{s}[\rho]$, greater insight into density scaling is gained by considering the link with particle number scaling in the reference system for the kinetic energy. We thus digress a little to define an auxiliary quantity used in this work, $T_{\zeta}[\rho]$, which we term the $\zeta$-kinetic energy. 
The Kohn-Sham kinetic energy is the kinetic energy of a ground-state $\mathrm{N}$-electron, $\mathrm{N}$-orbital noninteracting system that represents the true $N$-electron density. However, for some $N$-electron density $\rho(\mathbf{r})$, we can instead choose an $M$-electron noninteracting reference system with groundstate density $\rho(\mathbf{r}) / \zeta$, where $\zeta=N / M$. For integers $N$ and $M$, this corresponds to choosing an $M$-orbital reference system, e.g., in the neon atom, instead of a ten-orbital reference system, we could consider a six-orbital $1 s^{2} 2 s^{2} 3 s^{2}$ reference system (where $M=6, \zeta=5 / 3$ ), or even a two-orbital $1 s^{2}$ reference system (where $M=2, \zeta=5$ ). The $\zeta$-kinetic energy $T_{\zeta}[\rho]$ is then defined as $\zeta$ times the kinetic energy of the $M$-electron reference system, that is, for integer $M, N$,

$$
\begin{gathered}
T_{\zeta}[\rho]=\min _{\zeta \sum_{i}^{M} \phi_{i}^{2} \rightarrow \rho}-\frac{1}{2} \zeta \sum_{i}^{M}\left\langle\phi_{i}\left|\nabla_{i}^{2}\right| \phi_{i}\right\rangle, \\
\rho(\mathbf{r})=\zeta \sum_{i}^{M} \phi_{i}^{2}(\mathbf{r}),
\end{gathered}
$$

or more generally, when $M$ and $N$ are any number,

$$
\begin{aligned}
& T_{\zeta}[\rho]=\min _{\zeta \hat{\Gamma} \rightarrow \rho(\mathbf{r})} \zeta \operatorname{Tr}[\hat{\Gamma} \hat{T}], \\
& \rho(\mathbf{r})=\zeta \operatorname{Tr}[\hat{\Gamma} \hat{\rho}],
\end{aligned}
$$

$T_{\zeta}[\rho]$ is not an unfamiliar quantity. Important choices of $\zeta$ in this work are $\zeta=1(M=N)$ and $\zeta=N(M=1)$. In these limits, $T_{1}[\rho]=T_{s}[\rho]$, and $T_{N}[\rho]=-\frac{1}{2} \int \rho^{\frac{1}{2}}(\mathbf{r}) \nabla^{2} \rho^{\frac{1}{2}}(\mathbf{r}) d \mathbf{r}$, the von Weizsäcker kinetic energy $[18,19]$.

The relation between $T_{\zeta}[\rho]$ and the density scaling of $T_{s}[\rho]$ is made explicit through simple scaling relations, which we derive as follows. If $\zeta \rightarrow \kappa \zeta$, the number of particles $M$ in the reference system goes from $N / \zeta \rightarrow N /(\kappa \zeta)$, and if we simultaneously scale $\rho(\mathbf{r})$ by $\kappa$, then $M$ is unchanged by the combined two scaling procedures. So, we have relations

$$
\begin{gathered}
T_{\kappa \zeta}[\rho]=\kappa T_{\zeta}\left[\frac{\rho}{\kappa}\right], \\
T_{\zeta}[\rho]=\zeta T_{s}\left[\frac{\rho}{\zeta}\right],
\end{gathered}
$$

where Eq. (13) is just a specific case of Eq. (12) (with $\kappa$ $=1$ ), and is a scaling relation for the Kohn-Sham kinetic energy, since $T_{1}[\rho / \zeta]=T_{s}[\rho / \zeta]$. Thus, studying the behavior of $T_{\zeta}[\rho]$ with $\zeta$ is equivalent to studying the scaling behavior of $T_{s}[\rho]$, and we shall use this relation later in this work.

We finish by defining other energy quantities that appear in the Kohn-Sham version of density-functional theory. In Kohn-Sham theory, the energy functional is partitioned as

$$
E[\rho]=T_{s}[\rho]+J[\rho]+E_{\mathrm{xc}}[\rho]+\int \rho(\mathbf{r}) v(\mathbf{r}) d \mathbf{r} .
$$

The classical Coulomb repulsion $J[\rho]$ $=\frac{1}{2} \iint\left[\rho\left(\mathbf{r}_{1}\right) \rho\left(\mathbf{r}_{2}\right) / r_{12}\right] d \mathbf{r}_{1} d \mathbf{r}_{2}$, and $E_{\mathrm{xc}}[\rho]$ is the exchangecorrelation functional, which incorporates all nonclassical correlation effects. Its functional derivative, the exchangecorrelation potential $v_{\mathrm{xc}}(\mathbf{r})=\delta E_{\mathrm{xc}}[\rho] / \delta \rho(\mathbf{r})$ appears in the Kohn-Sham orbital equations. Analogously, using $T_{\xi}[\rho]$, we can write a partitioning of the energy

$$
E[\rho]=T_{\zeta}[\rho]+J[\rho]+E_{\zeta \times c}[\rho]+\int \rho(\mathbf{r}) v(\mathbf{r}) d \mathbf{r},
$$

where we have defined the $\zeta$-exchange-correlation energy $E_{\zeta \mathrm{xc}}[\rho]$, and its corresponding functional derivative $v_{\zeta \mathrm{xc}}(\mathbf{r})$ $=\delta E_{\zeta \mathrm{xc}}[\rho] / \delta \rho(\mathbf{r})$.

With the relevant quantities defined, we proceed to investigate the explicit density scaling behavior of $T_{s}[\rho]$ and $T[\rho]$ in the next sections.

\section{FORMAL STUDY OF DENSITY SCALING}

Here we consider the density scaling of the kinetic energy from a formal point of view, as a first step towards the goal of bounds and strict inequalities that can be used to construct new functionals. Formally, the density scaling behavior of the kinetic energies $T_{s}[\rho]$ and $T[\rho]$ is closely related to the properties of convexity and homogeneity. We start by defining these.

A functional $F[\rho]$ is convex in $\rho(\mathbf{r})$, when

$$
F\left[\zeta \rho_{1}+(1-\zeta) \rho_{2}\right] \leqslant \zeta F\left[\rho_{1}\right]+(1-\zeta) F\left[\rho_{2}\right],
$$

for $\zeta \leqslant 1$, and $\rho(\mathbf{r})$ in the domain of $F[\rho] . F[\rho]$ is homogeneous of order $\kappa$ in $\rho(\mathbf{r})$, when

$$
F[\zeta \rho]=\zeta^{\kappa} F[\rho] .
$$

If $F[\rho]$ is also smooth, or differentiable (in the sense described in Appendix A), then Eq. (17) implies

$$
\kappa F[\rho]=\int \frac{\delta F[\rho]}{\delta \rho(\mathbf{r})} \rho(\mathbf{r}) d \mathbf{r} .
$$

Note that it follows simply, if a functional is both homogeneous and convex, it must be homogeneous of order $\kappa \geqslant 1$.

How does this relate to the density scaling of the kinetic energy? We deal first with convexity. It is a simple exercise to show that the density-matrix definitions of $T_{s}[\rho]$ and $E[\rho]$ (see Sec. II) are convex functionals in $\rho(\mathbf{r})$, and a brief proof is given in Appendix A. Then, since the kinetic energy of a zero particle system must be zero by definition, i.e., $T_{s}[0]=0$, we have the simple density scaling inequality

$$
T_{s}[\zeta \rho] \geqslant \zeta T_{s}[\rho], \quad \zeta>1,
$$

where the inequality is reversed if $\zeta<1$. Equation (19) is not a very strong bound on the kinetic energy, although the von Weizsäcker functional, $-\frac{1}{2} \int \rho^{1 / 2}(\mathbf{r}) \nabla^{2} \rho^{1 / 2}(\mathbf{r}) d \mathbf{r}$, lies "on the limits" of the inequality (19). Note, however, that Eq. (19) holds for densities of any particle number.

For densities with integer numbers of particles, there exists a stronger inequality, proved by Lieb and Thirring [11]: 


$$
T_{s}[\rho] \geqslant(8 \pi)^{-2 / 3} K_{c} \int \rho^{5 / 3}(\mathbf{r}) d \mathbf{r},
$$

with $K_{c}=(3 / 5)\left(6 \pi^{2}\right)^{2 / 3}$, and where the same inequality holds for $T[\rho]$. Constraint (20) plays a key role in the proof of stability of fermionic matter. Equation (20) becomes an increasingly strong constraint on the kinetic energy, as the density increases. For example, for sufficiently large $\rho(\mathbf{r})$, the von Weizsäcker functional does not obey Eq. (20).

From the scaling relations (13) and (19), we can derive further conditions on the $\zeta$-kinetic energy $T_{\zeta}[\rho]$ :

$$
\begin{aligned}
& T_{\zeta}[\rho] \leqslant T_{\zeta^{\prime}}[\rho], \quad\left(\zeta \geqslant \zeta^{\prime} \mid \zeta, \zeta^{\prime} \geqslant 1\right), \\
& T_{\zeta}[\rho] \leqslant T_{\zeta^{\prime}}[\rho], \quad\left(\zeta \leqslant \zeta^{\prime} \mid \zeta, \zeta^{\prime} \leqslant 1\right) .
\end{aligned}
$$

For specific choices of $\zeta$, the above relations yield familiar bounds. For example, taking $\zeta=N$ and $\zeta^{\prime}=1$, we recover Harriman's result [20], that the von Weizsäcker kinetic energy is never larger than the Kohn-Sham kinetic energy; $T_{s}[\rho] \geqslant T_{N}[\rho]$. More generally, the inequalities (21) and (22) state that $T_{\zeta}[\rho]$ as a function of $\zeta$ has a maximum at $\zeta=1$ (the Kohn-Sham kinetic energy). In other words, choosing anything other than $N$ orbitals to represent the kinetic energy results in a $T_{\zeta}[\rho]$ that is less than $T_{s}[\rho]$. Since the Kohn-Sham kinetic energy is a lower bound to $T[\rho]$, the Kohn-Sham choice of $\zeta=1$ is the best choice of $\zeta$, as one recovers the maximum amount of the interacting kinetic energy $T[\rho]$.

We now discuss homogeneity of the kinetic energy. Homogeneous functionals occupy a special place in densityfunctional theory, as they obey the very simple scaling relation (18). It is also known that any functional which has, to all orders, well-defined functional derivatives that are strongly vanishing, can be expressed as a sum of homogeneous functionals [21]. A priori we would not expect the kinetic energy to be homogeneous, as both $T[\rho]$ and $T_{s}[\rho]$ have a complex dependence on $N$ which does not seem adequately described by a relation of the form (17). Despite this, using the one-electron density matrix as an intermediary, Liu and Parr [6] showed that, under conditions where the necessary functional derivatives exist,

$$
T_{s}[\rho]=\int \frac{\delta T_{s}[\rho]}{\delta \rho(\mathbf{r})} \rho(\mathbf{r}) d \mathbf{r} .
$$

We term this equation the Liu-Parr relation. From Eq. (17), it would follow naively that

$$
T_{s}[\zeta \rho]=\zeta T_{s}[\rho]
$$

and similarly for $T[\rho]$. Equation (24) states that $T[\rho]$ and $T_{s}[\rho]$ are homogeneous of order 1 in the density. We shall term it the kinetic-energy homogeneity relation.

Is Eq. (24) too good to be true? Clearly it is a very strong equality, which lies "on the limits" of the convexity of $T_{s}[\rho]$, and in fact, we immediately see that Eq. (24) does not agree with the Lieb-Thirring bound (20). It is too optimistic to assume that Eq. (24) follows from Eq. (23), and there are many ways to demonstrate the homogeneity relation (24) does not hold. These we summarize now.
Theorem. The kinetic energy homogeneity relation, Eq. (24), is false.

Proof. Equation (24), for sufficiently large $\rho(\mathbf{r})$, does not agree with the Lieb-Thirring bound (20), which requires the kinetic energy to contain at least one component of homogeneity $\kappa \geqslant 5 / 3$.

Comment. Note that the Lieb-Thirring bound was only derived for densities with integer numbers of particles, and thus we are restricting our attention to that class of densities in the above statement. For a more explicit proof, consider the following. Assume the homogeneity relation (24) is true. Define $N=\int \rho(\mathbf{r}) d \mathbf{r}$. Then from the scaling relation (13), remembering that $T_{s}[\rho]$ is $T_{1}[\rho]$, and $T_{s}[\zeta \rho]=\zeta T_{\zeta}[\rho]$, comparing with Eq. (24) yields

$$
T_{s}[\rho]=T_{\zeta}[\rho]
$$

Take, for example, $\zeta=N$, which corresponds to a choice of the von Weizsäcker kinetic energy for $T_{\zeta}[\rho]$. Equation (25) states that as a consequence of homogeneity, the Kohn-Sham kinetic energy is identical to the von Weizsäcker kinetic energy. There are numerous counterexamples to this, and so supposition (24) must be false. Note that since $T[\rho]$ $\geqslant T_{s}[\rho]$, and the equality is satisfied for one-electron systems, we have also proved that the homogeneity relation does not hold for $T[\rho]$.

If Eq. (24) does not hold, $T_{s}[\rho]$ and $T[\rho]$ cannot be considered to be homogeneous of order 1 in the density. What do we then make of the Liu-Parr relation, Eq. (23)? The essence of the matter lies in the fact that the kinetic-energy functionals $T[\rho]$ and $T_{s}[\rho]$ are not smooth [15]. For example, for the Kohn-Sham kinetic energy, when we increase the number of electrons (e.g., through density scaling) in a system with an integer number of particles, the extra density goes into new orbitals (by the Pauli principle), which, from the discrete eigenvalue spectrum of the orbitals, leads to nonsmooth behavior of $T_{s}[\rho]$.

As a consequence of nonsmoothness, the necessary functional derivatives of $T[\rho]$ and $T_{s}[\rho]$ do not exist everywhere, which is a necessary condition for the Liu-Parr proof. Instead, $\delta T_{s}[\rho] / \delta \rho(\mathbf{r})$ is in general undefined; we must specify the path $\sigma(\mathbf{r})$ used to define the derivative. Even if there exists some $\sigma(\mathbf{r})$ such that Eq. (23) holds, it does not follow that Eq. (24) is true. In view of the poor behavior of functional derivatives, in density-functional theory, we should be cautious when interpreting predicted homogeneities of other energy components [5].

However, this does not rule out there being a restricted set of densities $\mathcal{S}$, for which the kinetic energy $T[\rho]$ or $T_{s}[\rho]$ does satisfy a homogeneity relation (17). The conditions under which this can occur, and the implications for the behavior of the functional derivatives, are discussed in greater detail in Appendix A. For $\rho(\mathbf{r}) \in \mathcal{S}$, we might term the kinetic energy effectively homogeneous of order $\kappa$. An example of a set $\mathcal{S}$ is the set of uniform densities, where the ThomasFermi [22] functional $T[\rho]=K_{c} 2^{-2 / 3} \int \rho^{5 / 3}(\mathbf{r}) d \mathbf{r}$ is exact, and displays an effective homogeneity of $5 / 3$. Whether $T[\rho]$ or $T_{s}[\rho]$ exhibits an effective homogeneity in other classes of densities, is a question that can only be answered by computation, and is investigated in the next section. 


\section{NUMERICAL STUDY OF DENSITY SCALING}

Here we perform a numerical study of the density scaling of the Kohn-Sham kinetic energy $T_{s}[\rho]$, which is a tight lower bound to the true kinetic energy $T[\rho]$. To compute $T_{s}[\zeta \rho]$, we compute $T_{\zeta}[\rho]$ for several values of $\zeta$, for a given ab initio density, and then infer $T_{s}[\zeta \rho]$ from the scaling relation (13). In the first subsection, we introduce the generalized Kohn-Sham orbital equations, and ZhaoMorrison-Parr procedure, associated with calculating $T_{\xi}[\rho]$ from a given density.

In the second subsection, we present our computed results for the closed shell atomic systems $\mathrm{Be}, \mathrm{Ne}, \mathrm{Mg}$, Ar. We carry out a study of effective homogeneity, and also investigate whether $T_{\zeta}[\rho](\zeta>1)$ is a useful kinetic-energy functional.

\section{A. Methodology}

The Kohn-Sham orbital equations are the starting point for a Kohn-Sham density-functional calculation. We can derive a similar scheme involving $T_{\xi}[\rho]$, through the partitioning (15). Recall that $\zeta=N / M$. Henceforth, we are concerned only with $M, N$ integer, where we write down $M$ generalized Kohn-Sham orbital equations

$$
\begin{gathered}
{\left[-\frac{1}{2} \nabla^{2}+v(\mathbf{r})+v_{J}(\mathbf{r})+v_{\zeta \mathrm{xc}}(\mathbf{r})\right] \phi_{i}(\mathbf{r})=\epsilon_{i} \phi_{i}(\mathbf{r}),} \\
1 \leqslant i \leqslant M,
\end{gathered}
$$

with the density resolved as

$$
\rho_{0}(\mathbf{r})=(N / M) \sum_{i}^{M} \phi_{i}^{2}(\mathbf{r}) .
$$

Here, $v_{J}(\mathbf{r})$ is the Coulomb potential $v_{J}(\mathbf{r})=\int\left[\rho\left(\mathbf{r}_{2}\right) /\right.$ $\left.r_{12}\right] d \mathbf{r}_{2}$, and $v_{\zeta \mathrm{xc}}(\mathbf{r})$ is termed the $\zeta$-exchange-correlation potential (defined in Sec. II). For the closed-shell systems studied in this work, $\phi_{i}(\mathbf{r})=\phi_{i+1}(\mathbf{r})$, for odd $i$, i.e., the orbitals are paired. The full derivation of the orbital equations is not relevant here, and is left until Appendix B. However, we briefly elaborate on the $\zeta$-exchange-correlation potential. When $\zeta=1$, the above equations are the Kohn-Sham equations, that is $v_{1 \mathrm{xc}}(\mathbf{r})=v_{\mathrm{xc}}(\mathbf{r})$. In general, however, $v_{\zeta \mathrm{xc}}(\mathbf{r})$ is not the Kohn-Sham exchange-correlation potential associated with density $\zeta \rho(\mathbf{r})$. This is because, as mentioned earlier, the ground-state external potential changes under the operation of density scaling. Instead, $v_{\zeta \mathrm{xc}}(\mathbf{r})$ is the manybody effective local potential, that forces the scaled $M$-particle density [Eq. (27)] to reproduce the $N$-particle ground-state density, associated with $v(\mathbf{r})$. From Eqs. (14) and (15), we see that the $\zeta$-exchange-correlation potential is related to the usual Kohn-Sham exchange-correlation potential through

$$
v_{\zeta \mathrm{xc}}(\mathbf{r})=v_{\mathrm{xc}}(\mathbf{r})+\frac{\delta}{\delta \rho(\mathbf{r})}\left(T_{\zeta}[\rho]-T_{s}[\rho]\right) .
$$

To minimize $E[\rho]$, the orbital equations (26) are solved iteratively to find the ground-state density $\rho_{0}(\mathbf{r})$. If we choose $\zeta>1$, there are $M<N$ equations, which is enticing, because the computational effort is less than that required in the conventional Kohn-Sham scheme. In particular, there has been considerable interest in the case where $M=1$ (or 2, for closed shells), which corresponds to an "exact" extended Thomas-Fermi theory. In such a case, the orbital equation reduces to a differential equation for the density amplitude $\rho^{1 / 2}(\mathbf{r})[23,24]$.

In light of the above, we now digress briefly to ask the following: are the generalized Kohn-Sham equations, for $\zeta$ $>1$, a viable simplification of the Kohn-Sham procedure? This is equivalent to asking whether $T_{\xi}[\rho]$ is a suitable substitute for $T_{s}[\rho]$. There are two foreseeable obstacles. First, the residual $\zeta$-exchange-correlation energy $E_{\zeta \times \mathrm{c}}[\rho]$ in the partitioning (15) may be quite large. When $\zeta=1$ (conventional Kohn-Sham), we know the exchange-correlation component is small, but this may no longer be the case if $\zeta>1$, since the contribution from the kinetic-energy term $\delta / \delta \rho(\mathbf{r})\left(T_{\zeta}[\rho]-T_{s}[\rho]\right)$ may be quite large. The second obstacle is that $E_{\zeta \mathrm{xc}}[\rho]$ may simply be hard to approximate. By this we mean that the functional may be highly nonlocal and nonanalytic. The behavior of the functional derivative $v_{\zeta \mathrm{xc}}(\mathbf{r})$ will yield information on the behavior of $E_{\zeta \mathrm{xc}}[\rho]$.

The inversion of the generalized Kohn-Sham Eqs. (26) is the basis for our computation of $T_{\zeta}[\rho], v_{\zeta \mathrm{xc}}(\mathbf{r})$, and $T_{s}[\zeta \rho]$, from given input densities $\rho_{0}(\mathbf{r})$. The problem may be posed thus: given some density $\rho_{0}(\mathbf{r})$, we wish to find $M$ orbitals, for which we also need $v_{\zeta \times c}(\mathbf{r})$, a total of $M+1$ unknowns. We have the $M$ orbital equations, and the relation $\rho(\mathbf{r})$ $=\zeta \Sigma_{i}^{M} \phi_{i}^{2}(\mathbf{r})$. Solutions to this problem have been known for some time, and are summarized in van Leeuwen et al. [10]. We adopt the method of Zhao-Morrison-Parr (ZMP) [12], which is derived in Appendix B. Here, we note only that, for given integer $M$ orbitals in the reference system (and corresponding $\zeta=N / M$ ), we solve the $M$ ZMP orbital equations, which are just Eqs. (26), with $v_{\zeta \times c}$ replaced by the quantity

$$
v_{\zeta \mathrm{xc}}^{\lambda}(\mathbf{r})=\lambda \int \frac{\rho\left(\mathbf{r}_{2}\right)-\rho_{0}\left(\mathbf{r}_{2}\right)}{r_{12}} d \mathbf{r}_{2}-\frac{1}{N} \int \frac{\rho\left(\mathbf{r}_{2}\right)}{r_{12}} d \mathbf{r}_{2},
$$

where $\rho_{0}(\mathbf{r})$ is the input density, and $\rho(\mathbf{r})$ is the density yielded by the orbitals. When we take the solutions in the limit $\lambda \rightarrow \infty, v_{\zeta \mathrm{xc}}^{\lambda}(\mathbf{r}) \rightarrow v_{\zeta \mathrm{xc}}(\mathbf{r})$, and the orbitals $\phi_{i}(\mathbf{r})$ yield $T_{\zeta}[\rho]$ through Eq. (8).

In the ZMP method there are two technical difficulties which are relevant to the calculations in this work. First, in a finite basis set, $\rho(\mathbf{r})-\rho_{0}(\mathbf{r})$ can never vanish, and the ZMP orbital equations must be solved at a finite value of $\lambda$ to prevent divergent eigenvalues and potentials [25]. There will be arbitrariness in our results from the choice of $\lambda_{\text {opt }}$, but there is a range of $\lambda$ over which the kinetic energies and potentials we obtain are stable, and we choose $\lambda_{\text {opt }}$ in this region of stability. Note that in a finite basis set, as the number of orbitals $M$ in the reference system increases ( $\zeta$ decreases), the representation of the input density $\rho_{0}(\mathbf{r})$ improves, and we can use higher $\lambda_{\text {opt }}$ values.

The second difficulty is our need for suitable guess orbitals, to solve the ZMP orbital equations. Here we study only closed-shell spherical systems, and thus the basic criterion is that our set of $M$ guess orbitals yields an overall spherical guess density. A simple solution is to perform Hartree-Fock 


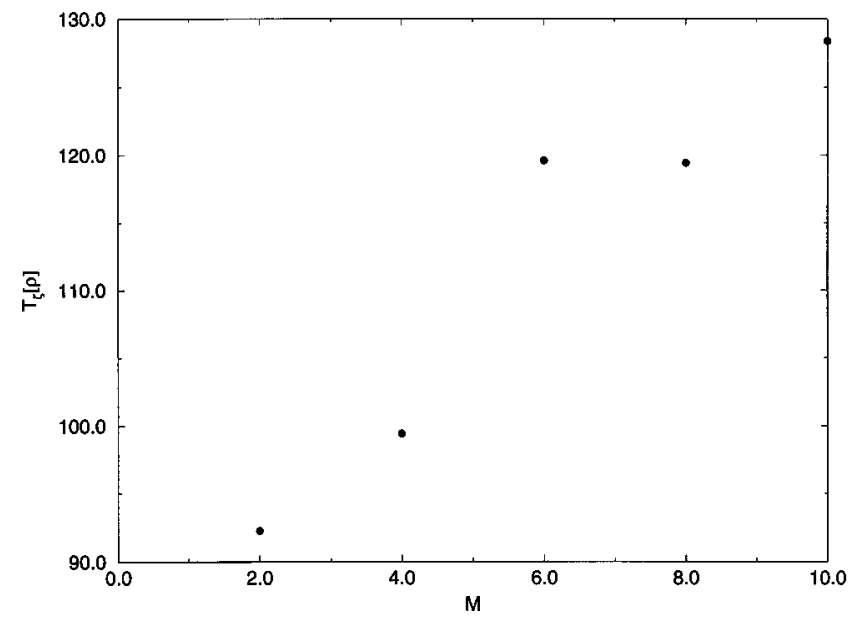

FIG. 1. The $\zeta$ kinetic energy $T_{\zeta}[\rho]$ (units of $E_{\mathrm{H}}$ ) against the number of orbitals in the reference system $M$, for $\mathrm{Ne}(\mathbf{)})$.

calculations on the systems to yield guess orbitals, choosing the lowest set (in the sense of the eigenvalue sum) of $M$ guess orbitals which yield a spherical guess density. For example, for the $M=6$ treatment of neon, we choose a $1 s^{2} 2 s^{2} 3 s^{2}$ guess configuration of neon Hartree-Fock orbitals. The Hartree-Fock guess orbitals will, of course, be spatially contracted from the solution orbitals. Again, as $M$ increases ( $\zeta$ decreases), we expect the suitability of our guess orbitals to improve.

Because of the various difficulties, in practice, the selfconsistent solution of the ZMP equations may not converge for all values of $\zeta$. When it does, the calculated kinetic energies $T_{\zeta}[\rho], T_{s}[\zeta \rho]$, and $v_{\zeta \mathrm{xc}}(\mathbf{r})$, will be accurate only to a few percent, with the accuracy decreasing as $\zeta \rightarrow 1$. Such calculations are still useful, however, as the qualitative behavior of the quantities will not be affected.

\section{B. Computations}

Using the Zhao-Morrison-Parr method, we computed $T_{\zeta}[\rho]$ and $v_{\zeta \mathrm{xc}}(\mathbf{r})$ for the closed shell atoms: beryllium, neon, magnesium, and argon, for integer $M$ and the associated values of $\zeta=N / M$. The input densities $\rho_{0}(\mathbf{r})$ were second-order Møller-Plesset $a b$ initio densities, calculated from CADPAC [26], using large Partridge-1 uncontracted $s p$ basis sets (Be 14s, Ne 14s9p, Mg 18s10p, Ar 17s12p) [27], with the densities "relaxed" such that they correspond to the density matrix used when evaluating derivatives of the MP2 energy. For $M<N$, we used $\lambda_{\text {opt }}=50$; for $M=N$, we used $\lambda_{\text {opt }}=200$. Corresponding $T_{s}[\zeta \rho]$ values were then calculated from $T_{\zeta}[\rho]$ via the scaling relation (13).

In Fig. 1, we plot $T_{\zeta}[\rho]$ against the number of orbitals $M$, for the neon atom. $T_{\zeta}[\rho]$ displays the expected behavior; it increases as $M$ increases ( $\zeta$ decreases) obeying condition (21). Similar behavior is observed for $T_{\zeta}[\rho]$ in the other atoms. Note that for $M=N-2$ (one less closed-shell orbital in the reference system), the corresponding value of $T_{\zeta}[\rho]$ is significantly less than $T_{s}[\rho]$. In general, $T_{\zeta}[\rho]$, for reference systems with fewer than $N$ orbitals, is a poor approximation to $T_{s}[\rho]$ and $T[\rho]$. In contrast, $T_{s}[\rho]$ is a good approximation to $T[\rho]$, with the difference being of the order of the correlation energy.
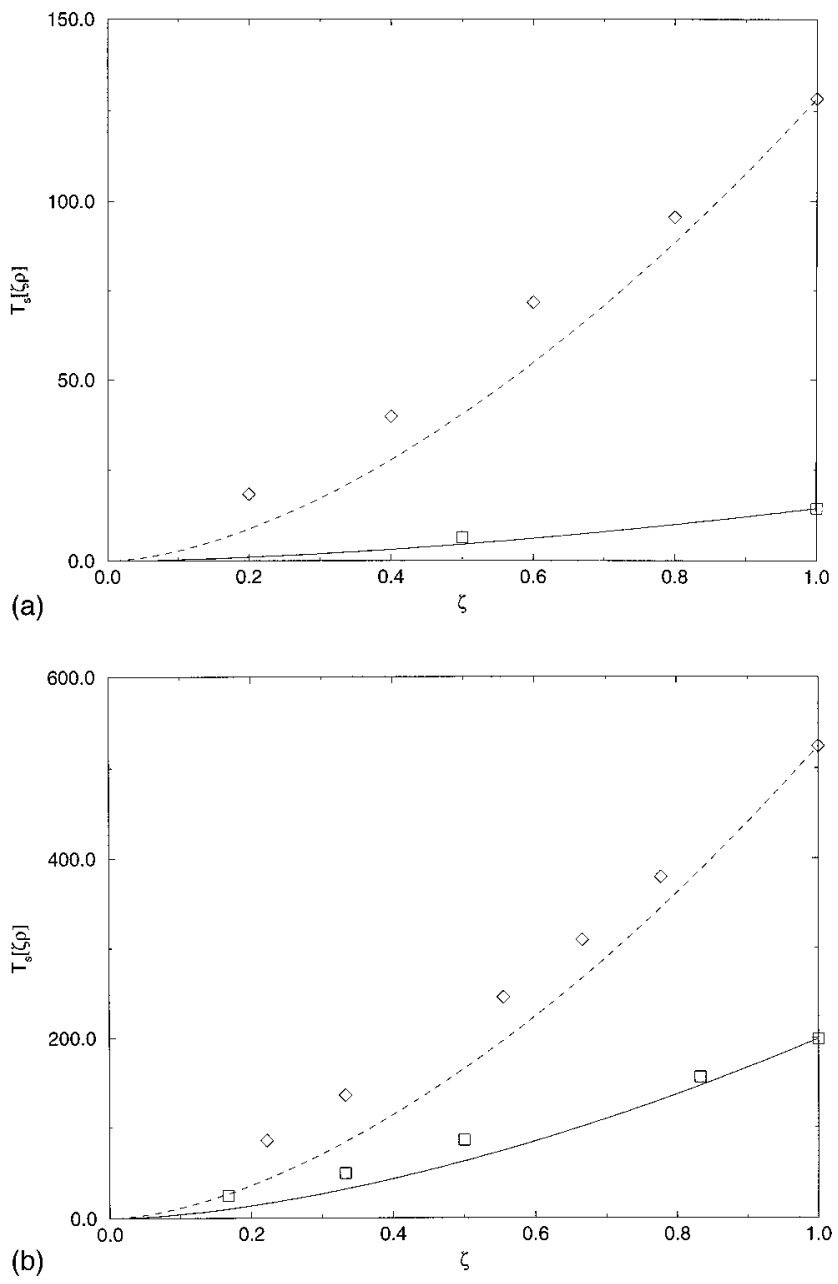

FIG. 2. (a) $T_{s}[\zeta \rho]$ (units of $E_{\mathrm{h}}$ ) against $\zeta$ in $\mathrm{Ne}(\diamond)$ and Be ( $\square$ ). The dashed line is the curve $\zeta^{5 / 3} T_{s}[\rho]$ for Ne, and the solid line is the same curve for Be. (b) $T_{s}[\zeta \rho]\left(E_{\mathrm{H}}\right)$ against $\zeta$ in $\operatorname{Ar}(\diamond)$ and $\mathrm{Mg}(\square)$. The dashed line is the curve $\zeta^{5 / 3} T_{s}[\rho]$ for Ar, and the solid line the same curve for $\mathrm{Mg}$.

In Figs. 2(a) and 2(b), we plot $T_{s}[\zeta \rho]$ against $\zeta$ in $\mathrm{Be}, \mathrm{Ne}$, and $\mathrm{Mg}$, Ar, respectively. We see that $T_{s}[\zeta \rho]$ obeys the scaling condition (19). In addition, the data points can be smoothly interpolated between the points by a polynomial of the form $C \zeta^{\kappa}$, indicative of effective homogeneity in $T_{s}[\rho]$. Taking $C=T_{1}[\rho]$ and $\kappa=5 / 3$, we plot the curve $\zeta^{5 / 3} T_{1}[\rho]$, for each system. This fits the energy data well, and demonstrates that there is much good physics in Thomas-Fermi theory, which predicts exactly a homogeneity of $\kappa=5 / 3$ in the kinetic energy. The effective homogeneity in $T_{s}[\rho]$ is also consistent with previous studies of effective homogeneity in Hartree-Fock energies [28]. We can optimize the exponent $\kappa$ of the fitted polynomial curve to the kinetic energy data in a least-squares sense, to find a better value for the effective homogeneity. The optimized $\kappa$ are given for the various systems in Table I. We expect similar effective homogeneities to hold over a wide range of atomic and molecular densities, though it remains to be seen whether these effective homogeneities are of predictive value. Note of course, that these effective homogeneities are not consistent with a naive interpretation of the Liu-Parr relation (23), which supports our analysis in the previous section. Since this manuscript was submitted, further numerical evidence 
TABLE I. Effective kinetic-energy homogeneities, $\kappa$, for some atoms. See Sec. IV.

\begin{tabular}{cc}
\hline \hline & $\kappa$ \\
\hline $\mathrm{Be}$ & 1.16 \\
$\mathrm{Ne}$ & 1.21 \\
$\mathrm{Mg}$ & 1.18 \\
$\mathrm{Ar}$ & 1.20 \\
\hline \hline
\end{tabular}

against a naive interpretation of the Liu-Parr relation has also appeared [29].

In Fig. 3, we plot the $\zeta$-exchange-correlation potential $v_{\zeta \mathrm{xc}}(\mathbf{r})$, for the neon atom. As the number of orbitals in the reference system increases to $N(\zeta \rightarrow 1)$, there is a decrease in magnitude of $v_{\zeta \mathrm{xc}}(\mathbf{r})$, reflecting the decreasing magnitude of the $\zeta$-exchange-correlation energy. For most $\zeta$, the $\zeta$-exchange-correlation potentials are dominated by the positive "bumps" which characterize the depletion of density in the atomic intershell regions, and yield the shell structure in the density $\rho(\mathbf{r})$ through the orbital Eqs. (26). As $\zeta \rightarrow 1$, the nodal structure of the density is better reproduced and the size of the "bumps" decreases, so that when $M=N(\zeta=1$, the Kohn-Sham case), the intershell bumps are barely visible. For large $\zeta$, the intershell bumps are so large that $v_{\zeta \mathrm{xc}}(\mathbf{r})$ is often a positive quantity. Far away, however, all the potentials die off with a $-1 / r$ tail. This can be proved to be the correct long-range decay law for the $\zeta$-exchange-correlation potential $v_{\zeta \mathrm{xc}}(\mathbf{r})$ [30]. Similar behavior is observed for $v_{\zeta \mathrm{xc}}(\mathbf{r})$ in the other atomic systems.

It seems that not only is the Kohn-Sham choice of reference system $(M=N, \zeta=1)$ a good choice, it is by far the best choice. This is because when $\zeta=1, T_{\zeta}[\rho]$ is a good approximation to $T[\rho]$, and the scaled exchange-correlation potential is (i) nonoscillatory, (ii) small in magnitude, and (iii) simple in structure. The failure of extended ThomasFermi type theories [8] can be attributed to the need to model the difficult potential $v_{N \mathrm{xc}}(\mathbf{r})$; nor is the problem made significantly easier as $M$ (restricted to be integer) is increased, until the critical point $M=N$. We thus conclude that the

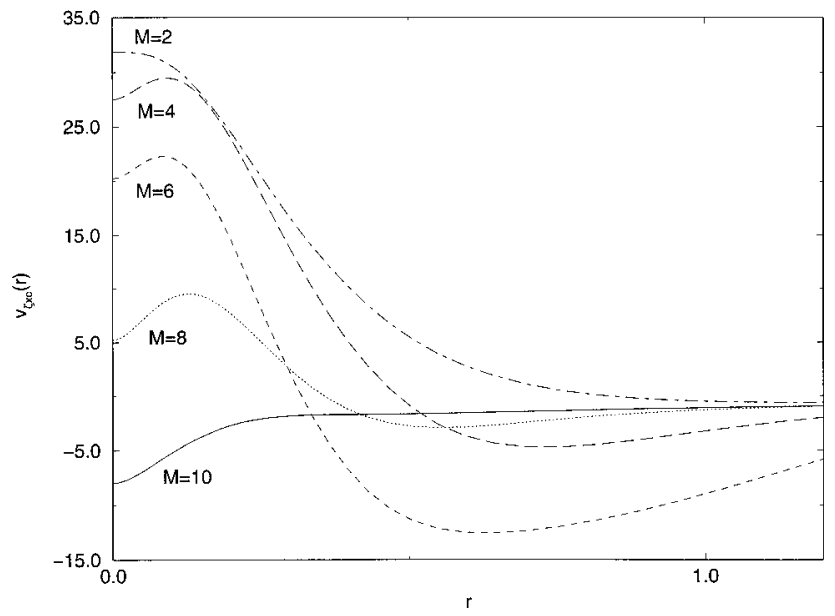

FIG. 3. The $\zeta$-exchange-correlation potential $v_{\zeta \times c}(\mathbf{r})$ (a.u.) against $r$ in the neon atom, for different $M$ (the number of orbitals in the reference system).
Kohn-Sham choice of $\zeta=1$, and the corresponding partitioning of the density into orbitals, is the only one to be useful computationally, because it contains the necessary physics. We stress, that in the search for simpler forms of the kineticenergy functional, it is important that the simplifications are themselves physically motivated, e.g., from spherical symmetry, or the natural separation of the density into core and valence regions, rather than purely of a mathematical nature (as for $T_{\zeta}[\rho]$ ).

\section{CONCLUSIONS}

In this work, we have undertaken the study of the density scaling of the kinetic energy, in particular, the Kohn-Sham kinetic energy $T_{s}[\rho]$ and the interacting kinetic energy $T[\rho]$. In the formal part of this work, several simple density scaling inequalities were derived from the convexity of the kineticenergy functional. We showed also that the homogeneity relation deduced by Liu and Parr [8] does not hold in practice, because the kinetic energy is a nonsmooth functional, and instead introduced the concept of an effective homogeneity for nonsmooth functionals.

In the numerical part of this work, we defined an intermediary quantity, the scaled kinetic energy, $T_{\zeta}[\rho]$, which is related to the discussion of the density scaling of $T_{s}[\rho]$. $T_{\zeta}[\rho]$ for $\zeta>1$, is also a simpler functional than $T_{s}[\rho]$, as it depends on fewer auxiliary orbitals. Using the method of Zhao-Morrison-Parr, we computed $T_{s}[\zeta \rho]$ via $T_{\zeta}[\rho]$ from the $a b$ initio densities of $\mathrm{Be}, \mathrm{Ne}, \mathrm{Mg}$, and Ar. The kinetic energies obeyed the scaling inequalities derived in this work. Moreover, the Kohn-Sham kinetic energy exhibited an effective homogeneity very close to $5 / 3$ in these systems.

By examining $T_{\zeta}[\rho]$, and the associated scaled exchangecorrelation potentials $v_{\zeta \mathrm{xc}}(\mathbf{r})$, we also showed that the KohnSham choice of $N$ orbitals to represent an $N$-particle system, is the only one to contain the necessary physics, and other models which partition the density in an unphysical fashion face considerable difficulties.

There are many open problems raised by this study. Can we extend this work to derive strong constraints on the kinetic-energy functional, which will help in the construction of functionals? Can we exploit the effective homogeneity of the kinetic energy? And how does the other nontrivial component of the energy, the exchange-correlation energy, scale? Further study is clearly needed.

\section{ACKNOWLEDGMENTS}

S. Liu, R. D. Daniel, and J. H-Y. Wei are thanked for interesting discussions on homogeneity theory. A. J. Cohen is thanked for help with figures.

\section{APPENDIX A}

\section{Proof of convexity}

In this section we prove convexity of the density-matrix definitions of the functionals used in this work.

Theorem. Define an energy functional $\Omega[\rho]$
$=\inf _{\hat{\Gamma} \rightarrow \rho} \operatorname{Tr}[\hat{\Omega} \hat{\Gamma}]$, where the infimum is over $N$-representable density matrices $\hat{\Gamma}$, and $\Omega$ is a linear operator. Then $\Omega[\rho]$ is convex in $\rho(\mathbf{r})$. 
Proof. Define a set $\mathcal{S}$ to be convex if for any elements $(\delta, \epsilon)$ in the set, $(1-\lambda) \delta+\lambda \epsilon$ is also in the set, where $\lambda$ $\leqslant 1$. Then the set of density matrices $\hat{\Gamma}$, characterized by spectral occupation numbers $f_{M i} \leqslant 1$ and $\Sigma_{M i} f_{M i}=1$, forms a convex set. Next, so long as $\hat{\Omega}$ is a linear operator, by acting on $\hat{\Gamma}$ it picks out a convex set. The infimum of the trace of this set, over the linear map $\hat{\Gamma} \rightarrow \rho$, is then a convex functional in $\rho(\mathbf{r})$.

Since both $\hat{T}$, and $\hat{T}+\hat{V}$ are linear operators, it follows that $T_{s}[\rho]$ and $E[\rho]-\int \rho(\mathbf{r}) v(\mathbf{r}) d \mathbf{r}$ are convex functionals in $\rho(\mathbf{r})$. Note that this does not resolve the long-standing question of the convexity of the ground-state $E$ as a function of $N$. Essentially, this is because we have not proved the equivalence of the density-matrix and wave-function definitions of the ground-state $E$ at integer $N$. Note also that we have used the infimum in the above proof for greater rigor, but in this work we have generally assumed the existence of a minimum.

\section{Functional derivatives}

Here we discuss aspects of functional derivatives relevant to this work. We begin by reviewing the definition of the functional derivative, and define smooth and nonsmooth behavior in functionals. Then we discuss the homogeneity of nonsmooth functionals.

Define a variation $\delta \rho(\mathbf{r})=\epsilon \sigma(\mathbf{r})$, where $\sigma(\mathbf{r})$ indicates the path (direction) of the variation, and $\epsilon$ is some small positive number. Then the functional derivative $\delta F[\rho] / \delta \rho(\mathbf{r})$ of a functional $F[\rho]$ is defined in the limit $\epsilon$ $\rightarrow 0_{+}$, through

$$
\delta F=\int \frac{\delta F[\rho]}{\delta \rho(\mathbf{r})} \delta \rho(\mathbf{r}) d \mathbf{r} .
$$

It follows simply, that

$$
\left[\frac{\partial F}{\partial \epsilon}\right]_{\epsilon=0}=\int \frac{\delta F[\rho]}{\delta \rho(\mathbf{r})} \sigma(\mathbf{r}) d \mathbf{r} .
$$

If $\delta F[\rho] / \delta \rho(\mathbf{r})$ exists, and is independent of $\sigma(\mathbf{r})$, then $\delta F[\rho] / \delta \rho(\mathbf{r})$ corresponds to a total derivative, and $F[\rho]$ is said to be smooth, or differentiable. Correspondingly, if the functional derivative depends on the path (direction) $\sigma(\mathbf{r})$, we have $\delta F[\rho, \sigma] / \delta \rho(\mathbf{r})$, which is a directional derivative, and $F[\rho]$ is nonsmooth at the point where the derivative is taken. This is analogous to the nonsmoothness of functions, which occurs at points where the left and right derivatives do not match.

If a nonsmooth functional $F[\rho]$ is effectively homogeneous of order $\kappa$ for a density $\rho \in \mathcal{S}$, what does this mean for the functional derivative $\delta F[\rho, \sigma] / \delta \rho(\mathbf{r})$ ? This question is raised in Sec. III. For such $F[\rho]$ and $\rho \in \mathcal{S}$, then $F[\zeta \rho]$ $=\zeta^{\kappa} F[\rho]$, and $\partial / \partial \zeta(F[\zeta \rho])=\kappa \zeta^{\kappa-1} F[\rho]$. Taking $\zeta=1$, and writing out the derivative with respect to $\zeta$ explicitly, we have

$$
\frac{\partial F}{\partial \zeta}=\lim _{d \zeta \rightarrow 0} \frac{F[\rho+\rho d \zeta]-F[\rho]}{d \zeta} .
$$

Comparing Eqs. (A2) and (A3), we identify $\epsilon=d \zeta / \gamma$ and $\sigma(\mathbf{r})=\gamma \rho(\mathbf{r})$ (i.e., variations along the density scaling path, where $\gamma$ is an arbitrary number, whose sign is such that $\epsilon$ is positive). We can then relate $F[\rho]$ to $\delta F[\rho, \sigma] / \delta \rho(\mathbf{r})$ :

$$
\kappa F[\rho]=\int d \mathbf{r} \rho(\mathbf{r})\left[\frac{\delta F[\rho, \sigma]}{\delta \rho(\mathbf{r})}\right]_{\sigma(\mathbf{r})=\gamma \rho(\mathbf{r})} .
$$

This is analogous to Eq. (18), and defines effective homogeneity for nonsmooth functionals. Note that along the path $\sigma(\mathbf{r})=\gamma \rho(\mathbf{r})$, a nonsmooth functional which obeys Eq. (A4) behaves smoothly.

\section{APPENDIX B}

Here we derive the generalized Kohn-Sham orbital equations, and discuss the Zhao-Morrison-Parr method for inverting them, as used in Sec. IV, in the numerical study of density scaling.

\section{The generalized Kohn-Sham equations}

The generalized Kohn-Sham equations are the natural framework to compute the $\zeta$-kinetic energy. We consider only the case for $M, N$ integer (and corresponding $\zeta$ $=N / M)$.

At the ground-state density $\rho_{0}(\mathbf{r})$ associated with an external potential $v(\mathbf{r})$, the interacting energy functional $E[\rho]$ satisfies an Euler equation [31]. Using the partitioning (15) for $E[\rho]$, this is written as

$$
\frac{\delta}{\delta \rho(\mathbf{r})}\left[T_{\zeta}[\rho]+J[\rho]+E_{\zeta \mathrm{xc}}[\rho]+\int \rho(\mathbf{r}) v(\mathbf{r}) d \mathbf{r}\right]_{\rho=\rho_{0}}-\mu=0
$$

where $\mu$ is the Lagrange multiplier associated with the constraint of constant particle number, and $v(\mathbf{r})$ is held fixed. Next, define a noninteracting $M$-particle reference system with density $\rho(\mathbf{r}) / \zeta$, in an effective potential $v_{\text {eff }}(\mathbf{r})$, with energy $T_{\zeta}[\rho] / \zeta+\int \rho(\mathbf{r}) v_{\text {eff }}(\mathbf{r}) / \zeta d \mathbf{r}$, and which minimizes at density $\rho(\mathbf{r})=\rho_{0}(\mathbf{r})$ [when the reference system density is $\left.\rho_{0}(\mathbf{r}) / \zeta\right]$. The Euler equation for this system is (multiplying all quantities by $\zeta$ ),

$$
\frac{\delta}{\delta \rho(\mathbf{r})}\left[T_{\zeta}[\rho]+\int \rho(\mathbf{r}) v_{\mathrm{eff}}(\mathbf{r})\right]_{\rho=\rho_{0}}-\mu_{\zeta}=0 .
$$

Equating the functional derivatives (B1) and (B2), and equating chemical potentials, identifies the effective potential of the reference system as

$$
v_{\mathrm{eff}}(\mathbf{r})=v_{J}(\mathbf{r})+v_{\zeta \mathrm{xc}}(\mathbf{r})+v(\mathbf{r}) .
$$

Finally, at the ground state of the reference system, the $M$ reference system orbitals $\phi_{i}(\mathbf{r})$ satisfy $\sum_{i}^{M} \phi_{i}^{2}(\mathbf{r})=\rho_{0}(\mathbf{r}) / \zeta$, and are eigenfunctions of the orbital equations

$$
\left[-\frac{1}{2} \nabla^{2}+v_{\mathrm{eff}}(\mathbf{r})\right] \phi_{i}(\mathbf{r})=\epsilon_{i} \phi_{i}(\mathbf{r}) .
$$

Substituting the expression for the effective potential (B3) in Eq. (B4), we arrive at the generalized Kohn-Sham Eqs. (26). 


\section{The Zhao-Morrison-Parr method}

The Zhao-Morrison-Parr method [12] centers on the Levy-Perdew [32] constrained search definition of the KohnSham kinetic energy [see Eq. (4)]. We modify the ZMP method to handle the $\zeta$-kinetic energy $T_{\zeta}[\rho]$ and related quantities, for which the corresponding constrained search (for $M, N$ integer) is Eq. (8).

Levy and Perdew [32], showed that we can enforce the constraint of fixed density in the energy minimization (8) through a Lagrange constraint functional $\Lambda[\rho]$. Then, we have the Lagrangian $\Omega\left[\left\{\phi_{i}\right\}\right]$, expressed as

$$
\Omega\left[\left\{\phi_{i}\right\}\right]=\zeta \sum_{i}^{M}\left\langle\phi_{i}\left|-\frac{1}{2} \nabla_{i}^{2}\right| \phi_{i}\right\rangle+\Lambda[\rho] .
$$

Minimizing explicitly with respect to the orbitals $\phi_{i}(\mathbf{r})$, with the usual diagonal orthonormality constraints associated with $\epsilon_{i}$, yields the orbital equations

$$
\left[-\frac{1}{2} \nabla^{2}+\frac{\delta \Lambda[\rho]}{\delta \rho(\mathbf{r})}\right] \phi_{i}(\mathbf{r})=\epsilon_{i}(\mathbf{r}) \phi_{i}(\mathbf{r}) .
$$

Comparison with Eq. (B4) yields the effective potential as the functional derivative of the constraint, $v_{\text {eff }}(\mathbf{r})$ $=\delta \Lambda[\rho] / \delta \rho(\mathbf{r})$.

Zhao, Morrison, and Parr [12] chose the following explicit form for the Lagrange constraint functional $\Lambda[\rho]$,

$$
\begin{aligned}
\Lambda[\rho]= & J[\rho]+\int \rho(\mathbf{r}) v(\mathbf{r}) \\
& +\lambda\left[\frac{1}{2} \iint \frac{\left[\rho\left(\mathbf{r}_{2}\right)-\rho_{0}\left(\mathbf{r}_{2}\right)\right]\left[\rho\left(\mathbf{r}_{1}\right)-\rho_{0}\left(\mathbf{r}_{1}\right)\right]}{r_{12}} d \mathbf{r}_{1} d \mathbf{r}_{2}\right] \\
& -\frac{1}{N} J[\rho],
\end{aligned}
$$

where $\rho(\mathbf{r})$ is the density yielded by the orbitals $\rho(\mathbf{r})$ $=\zeta \Sigma_{i}^{M} \phi_{i}^{2}(\mathbf{r}), \rho_{0}(\mathbf{r})$ is the input density, and $v(\mathbf{r})$ is the ground-state external potential associated with $\rho_{0}(\mathbf{r})$. Note that the double integral, with a dependence on $\rho_{0}(\mathbf{r})$, is the only actual constraint term on the density. Then, since at the solution point, $\rho(\mathbf{r})=\rho_{0}(\mathbf{r})$ and the kernel of the double integral vanishes, we must take the solution point at the limit $\lambda \rightarrow \infty$. Functionally differentiating $\Lambda[\rho]$, and comparing with the $v_{\text {eff }}(\mathbf{r})$ in the orbital Eqs. (B4) and (26), yields $v_{\zeta \mathrm{xc}}(\mathbf{r})=\lim _{\lambda \rightarrow \infty} v_{\zeta \mathrm{xc}}^{\lambda}(\mathbf{r}), \quad$ where $\quad v_{\zeta \mathrm{xc}}^{\lambda}(\mathbf{r})=\lambda \int\left[\rho\left(\mathbf{r}_{2}\right)\right.$ $\left.-\rho_{0}\left(\mathbf{r}_{2}\right) / r_{12}\right] d \mathbf{r}_{2}-1 / N \int\left[\rho\left(\mathbf{r}_{2}\right) / r_{12}\right] d \mathbf{r}_{2}$.

This is now an explicit computational method to invert from $\rho_{0}(\mathbf{r})$ to $v_{\zeta \mathrm{xc}}(\mathbf{r})$, and thus to $\phi_{i}(\mathbf{r})$ and $T_{\zeta}[\rho]$. Solve the ZMP orbital equations [Eq. (26) with $v_{\zeta \mathrm{xc}}(\mathbf{r})$ replaced by $\left.v_{\zeta \mathrm{xc}}^{\lambda}(\mathbf{r})\right]$, for values of $\lambda$, and take the limit $\lambda \rightarrow \infty$ to recover the physical $\phi_{i}(\mathbf{r})$ and related quantities. There are, however, technical problems with this method, which are discussed in Sec. IV.
[1] P. Hohenberg and W. Kohn, Phys. Rev. 136, B864 (1964).

[2] W. Kohn and L. J. Sham, Phys. Rev. 140, A1133 (1965).

[3] M. Levy and J. P. Perdew, Phys. Rev. A 32, 2010 (1985).

[4] J. P. Perdew and K. Burke, Int. J. Quantum Chem. 57, 309 (1996).

[5] R. G. Parr and S. B. Liu, Chem. Phys. Lett. 276, 164 (1997).

[6] S. B. Liu and R. G. Parr, Chem. Phys. Lett. 278, 341 (1997).

[7] N. H. March, Electron Density Theory of Atoms and Molecules (Academic Press, New York, 1991).

[8] E. K. U. Gross and R. M. Dreizler, Density Functional Theory (Springer-Verlag, Berlin, 1990).

[9] W. Yang, Phys. Rev. A 34, 4575 (1986).

[10] R. van Leeuwen, O. V. Gritsenko, and E. J. Baerends, in Topics in Current Chemistry 180, edited by R. F. Nalewajski (Springer-Verlag, Berlin, 1996).

[11] E. H. Lieb and W. E. Thirring, Phys. Rev. Lett. 35, 687 (1975).

[12] Q. Zhao, R. C. Morrison, and R. G. Parr, Phys. Rev. A 50, 2138 (1994).

[13] M. Levy, Proc. Natl. Acad. Sci. USA 76, 6062 (1979).

[14] E. H. Lieb, Int. J. Quantum Chem. 24, 243 (1983).

[15] J. P. Perdew, R. G. Parr, M. Levy, and J. L. Balduz, Jr., Phys. Rev. Lett. 49, 1691 (1982).

[16] P. A. M. Dirac, The Principles of Quantum Mechanics, 3rd ed. (Oxford University Press, Oxford, 1947).

[17] N. D. Mermin, Phys. Rev. 137, A1441 (1965).

[18] C. F. von Weizsäcker, Z. Phys. 96, 431 (1935)

[19] A. Theophilou, J. Phys. C 12, 5419 (1979).

[20] J. E. Harriman, in Density Matrices and Density Functionals, edited by R. Erdahl and V. H. Smith (Reidel, Dordrecht, 1987).
[21] S. B. Liu and R. G. Parr, Phys. Rev. A 55, 1792 (1997).

[22] L. H. Thomas, Proc. Cambridge Philos. Soc. 23, 542 (1927); E. Fermi, Z. Phys. 48, 73 (1928). It is interesting to note that in [6], Liu and Parr quote some recent work of N. H. March, Phys. Rev. A 56, 1025 (1997), which they claim shows that the Thomas-Fermi functional derivative may be considered homogeneous of order 0 . Of course this is suspicious, and a closer examination of March's paper reveals that the class of densities considered by March cannot be dense enough to admit the operation of density scaling. In other words, March's work shows that the Thomas-Fermi functional derivative, which is homogeneous of order $2 / 3$ may be equated with an expression that appears homogeneous of order 0 for certain densities, but this equality is not preserved under the operation of density scaling.

[23] G. Hunter, in Density Matrices and Density Functionals, edited by R. Erdahl and V. H. Smith, Jr. (Reidel, Dordrecht, 1987).

[24] M. Levy, J. P. Perdew, and V. Sahni, Phys. Rev. A 30, 2745 (1984).

[25] D. J. Tozer, V. E. Ingamells, and N. C. Handy, J. Chem. Phys. 105, 9200 (1997).

[26] R. D. Amos, I. L. Alberts, J. S. Andrews, S. M. Colwell, N. C. Handy, D. Jayatilaka, P. J. Knowles, R. Kobayashi, G. J. Laming, A. M. Lee, P. E. Maslen, C. W. Murray, P. Palmieri, J. E. Rice, E. D. Simandiras, A. J. Stone, M-D. Su, and D. J. Tozer, CADPAC6.0, The Cambridge Analytic Derivatives Package, Cambridge, UK, 1995.

[27] H. Partridge, J. Chem. Phys. 87, 6643 (1987); 90, 1043 (1989). 
[28] K. Ruedenberg, J. Chem. Phys. 66, 375 (1977).

[29] D. P. Joubert, Chem. Phys. Lett. 288, 338 (1998).

[30] G. K-L. Chan (unpublished).

[31] R. G. Parr and W. Yang, Density Functional Theory of Atoms and Molecules (Oxford University Press, Oxford, 1989).

[32] M. Levy and J. P. Perdew, in Density Functional Methods in Physics, edited by R. M. Dreizler and J. da Providencia (Plenum, New York, 1985). 